8th AIAA Multidisciplinary

Design Optimization Specialist Conference (MDO),

AIAA2012-1427. American Institute of

Aeronautics and Astronautics (AIAA), Honolulu, HA,

USA, 23-26 April 2012. doi:10.2514/1427.

\title{
An Investigation of Higher-order Multi-objective Optimisation for 3D Aerodynamic Shape Design
}

\author{
Timoleon Kipouros, Tiziano Ghisu† Geoffrey T. Parks ${ }^{\ddagger}$ \\ Department of Engineering, Cambridge University, Cambridge CB2 1PZ, United Kingdom \\ and A. Mark Savill ${ }^{\S}$ \\ Cranfield University, Cranfield MK43 OAL, United Kingdom
}

\begin{abstract}
We investigate the performance of different variants of a suitably tailored Tabu Search optimisation algorithm on a higher-order design problem. We consider four objective functions to describe the performance of a compressor stator row, subject to a number of equality and inequality constraints. The same design problem has been previously investigated through single-, bi- and three-objective optimisation studies. However, in this study we explore the capabilities of enhanced variants of our Multi-objective Tabu Search (MOTS) optimisation algorithm in the context of detailed 3D aerodynamic shape design. It is shown that with these enhancements to the local search of the MOTS algorithm we can achieve a rapid exploration of complicated design spaces, but there is a trade-off between speed and the quality of the trade-off surface found. Rapidly explored design spaces reveal the extremes of the objective functions, but the compromise optimum areas are not very well explored. However, there are ways to adapt the behaviour of the optimiser and maintain both a very efficient rate of progress towards the global optimum Pareto front and a healthy number of design configurations lying on the trade-off surface and exploring the compromise optimum regions. These compromise solutions almost always represent the best qualitative balance between the objectives under consideration. Such enhancements to the effectiveness of design space exploration make engineering design optimisation with multiple objectives and robustness criteria ever more practicable and attractive for modern advanced engineering design. Finally, new research questions are addressed that highlight the trade-offs between intelligence in optimisation algorithms and acquisition of qualitative information through computational engineering design processes that reveal patterns and relations between design parameters and objective functions, but also speed versus optimum quality.
\end{abstract}

\section{Introduction}

$\mathrm{M}$ ODERN Computational Engineering Design poses considerable challenges to and imposes demanding requirements on the available state-of-the-art optimisation algorithms. In order to study a realistic aerodynamic design problem we need to consider each of the most important flow metrics as individual objective functions subject to the appropriate physical and geometric constraints. ${ }^{[15}[19]$ These objectives are often in conflict, so the more of them the designer considers during a computational design task, the greater the insight and understanding of the specific design problem gained ${ }^{16}$ In this spirit, we investigate the aerodynamic design of $3 \mathrm{D}$ blades for axial compressors from a four-objective optimisation viewpoint, in order to assess the efficacy of such advanced techniques in engineering design. Previously, Harvey et al. 11 have studied this problem from a single-objective perspective, and Kipouros et al ${ }^{[15] 16]}$ presented bi-objective and three-objective studies of the same design problem. Multi-objective optimisation is found to be a more appropriate approach to complicated engineering design problems, a finding supported by Sasaki et al $l^{[18}$

\footnotetext{
*Research Associate, Cambridge Engineering Design Centre, Member.

${ }^{\dagger}$ Research Associate, Cambridge Engineering Design Centre .

${ }^{\ddagger}$ Senior Lecturer, Cambridge Engineering Design Centre.

$\S$ Professor, Department of Power and Propulsion, Senior Member.
} 
Compromise optimum designs are found to exhibit robust behaviour over a range of crucial flow metrics, and hence offer more stable performance with respect to the overall efficiency of the designed engineering system.

However, the need in detailed aerodynamic design for flow metric evaluations of sufficient accuracy is also

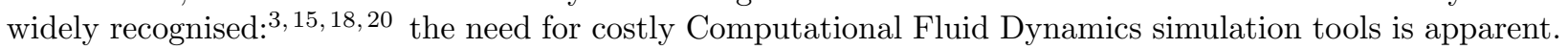
At the same time, engineering systems can only be flexibly and accurately modelled with a relatively large number of design parameters. All these factors contribute to making computational aerodynamic design optimisation problems challenging and, as a consequence, the performance of the optimisation algorithms used becomes an important factor in determining the viability of such techniques in real-world engineering design.

Another important characteristic that modern engineering design systems should exhibit is the ability to handle robustness criteria. The computational cost of design evaluation increases substantially when any of the design objectives requires the evaluation of robustness metrics, such as the mean and variance of the system performance with respect to uncertainties described by particular probability density functions $!^{5}$ This again places importance in the efficiency of the optimisation routine guiding the search.

In this study we investigate the performance of two different variants of the Multi-objective Tabu Search (MOTS) toolkit. The first is the PRMOTS variant, which has been presented by Jaeggi et al $\frac{13}{1}$ and adapted and applied by Kipouros et al $\frac{14}{14}$ to a real-world bi-objective optimisation problem. This implementation of MOTS is based on the path relinking principles proposed by Glover.10 The aim of this approach is to identify the design parameters associated with low (assuming minimisation) objective function solutions, and to reduce the dimensionality of the design space at each optimisation step by prioritising these.

The second variant, PCMOTS, has been developed and presented by Ghisu et al., 6 , 7 who applied it successfully to a number of test functions taken from the ZDT ${ }^{21}$ and $\mathrm{DTLZ}^{2}$ families and to a real-world aerodynamic test case, the preliminary design optimisation of the compressor core of an aero-engine. This implementation is based on Principal Components' Analysis, and the aim is to identify an optimal reorientation of the design parameters and to prioritise the most energetic of these rotated design parameters. The result is a more efficient search process that requires fewer design evaluations to find high quality solutions, since a reduction to the dimensionality of the design space is achieved.

\section{Description of the Four-Objective Optimisation Test Case}

We consider the detailed aerodynamic design of axial compressor blade rows by reducing flow separation and the general and secondary losses that develop through the row. The four objective metrics are blockage, entropy generation rate, profile losses and endwall losses - detailed definitions and descriptions of the modelling of these metrics can be found in Kipouros $\frac{16}{16}$ The mass flow rate is treated as an equality constraint, and the mass averaged flow turning, the minimum radius of the leading edge, and the tip clearance of the blade are considered as inequality constraints. The objective functions, equation 10 are normalised and include penalty function terms to handle the aerodynamic and geometric constraints.

$$
\begin{aligned}
f_{i}= & \frac{M_{i}}{M_{i, 0}}+250\left(1-\frac{\dot{m}}{\dot{m}_{0}}\right)^{2}+0.4 \max ^{2}\left(0,1-\frac{R_{L E}}{R_{L E, 0}}\right) \\
& +500 \max ^{2}\left(0,1-\frac{\Delta \theta}{\Delta \theta_{0}}\right)+0.5 \max ^{2}\left(0,1-\frac{C}{C_{l i m}}\right)
\end{aligned}
$$

In equation 1, $M$ represents any of the objective metrics, $\dot{m}$ is the mass flow rate, $R_{L E}$ is the minimum radius of the leading edge of the blade, $\Delta \theta$ is the mass-averaged flow turning, and $C$ measures the tip clearance of the blade. The zero subscripts identify the equivalent quantities for the datum blade geometry, the initial design in the optimisation.

The geometry is parameterised using a Partial Differential Equation approach, 1 giving a compact but flexible representation of the design, in a design vector comprising 26 variables. It should be noted here though that these parameters do not reflect direct geometrical characteristics (thickness, chord length, etc.) or any other engineering properties.

Both variants of the Multi-Objective Tabu Search algorithm have been tuned with the same parameter 
settings, in order to be able to assess directly the significance of their corresponding search enhancements.

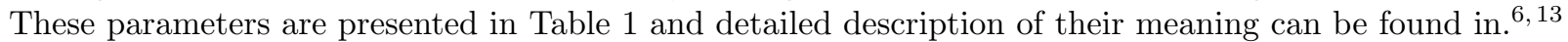

Table 1. Tabu Search Parameter Settings

\begin{tabular}{lll}
\hline Parameter & Description & Value \\
\hline$n \_s t m$ & Size of STM & 25 \\
$n \_r e g i o n s$ & Divide search space into n_var $*$ n_regions regions & 4 \\
intensify & Perform intensification when i_local = intensify & 35 \\
diversify & Perform diversification when i_local = diversify & 75 \\
restart & Reduce step sizes and restart when i_local = restart & 95 \\
$S S$ & Initial step sizes (as \% of variable range) & $1 \%$ \\
SSRF & Step sizes are multiplied by this factor at restart & 0.5 \\
$n \_s a m p l e$ & Number of points randomly sampled & 8 \\
select_interval & Perform variable selection every select_interval iterations & 20 \\
$n \_s e l e c t e d$ & Number of active design variables & $\frac{1}{2} n \_v a r$ \\
\hline
\end{tabular}

\section{Effects of Multi-objective Optimisation in Engineering Design}

Aerodynamic design problems are challenging tasks to tackle and combine complicated and highly nonlinear characteristics. There are many conflicting metrics that define and control the overall efficiency of a designed aerodynamic system. Figure 1 presents some optimum configurations for the aerodynamic design of an axial compressor blade row. The performance of different optimisation strategies on this difficult, real-world aerodynamic design application has been investigated in a succession of studies.

First, a single-objective study was performed by Harvey et al. $\frac{11}{11}$ considering blockage as the only objective function, subject to the same set of constraints as expressed in Eq. 1. Next, a bi-objective optimisation case, considering blockage and entropy generation rate as objectives, was investigated $\frac{15}{15}$ leading to a threeobjective case, with profile and endwall losses and blockage as the objectives ${ }^{[16]}$ We have now extended these studies to a four-objective case in which blockage, profile and endwall losses, and entropy generation rate are all to be minimised.

The richness of the design space for this application is readily apparent from the variation in the geometrical characteristics of the various optimum designs. However, the optimum design from the four-objective study appears to combine and blend geometrical characteristics identified in optimum designs from each of the previous studies. This observation justifies the fact that a complete investigation of this particular design problem has been achieved.

Figure 2 illustrates the 4D trade-off surface found using the PRMOTS variant of our optimisation algorithm. The complexities of the design space are clear with two disjoint regions forming the Pareto front.

\section{Effects and Assessment of Optimisation Intelligence in 3D Aerodynamic Shape Design}

Practical engineering design problems require a large number of design parameters in order to model design geometry accurately and flexibly enough. As consideration of multiple objectives inevitably increases the proportion of the design space in which optimal solutions are to be found, reducing the dimensionality of the design space can significantly improve the tractability of multi-objective design optimisation problems. In order to achieve this, sophisticated parameter selection techniques are required. In this work, we deploy the PCMOTS variant of our optimisation algorithm, which is equipped with the Principal Components' Analysis technique for the selection of the most active design parameters in an optimal orientation of the design space.$^{7}$ Ghisu et al. showed that using this technique for the preliminary design of an aero-engine core compression system the computational demands of the optimisation process reduce dramatically without compromising the quality of the trade-off surface found. 


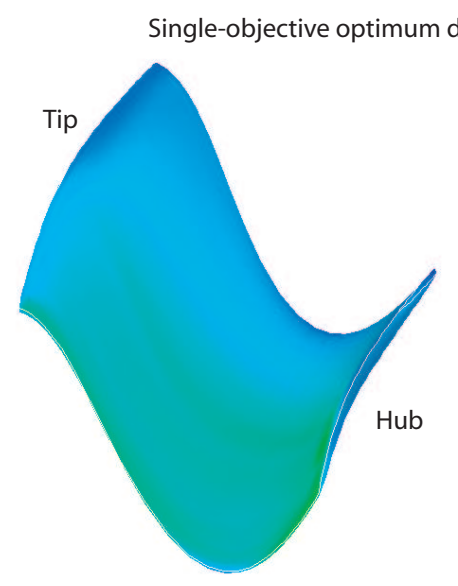

Bi-objective compromise optimum design
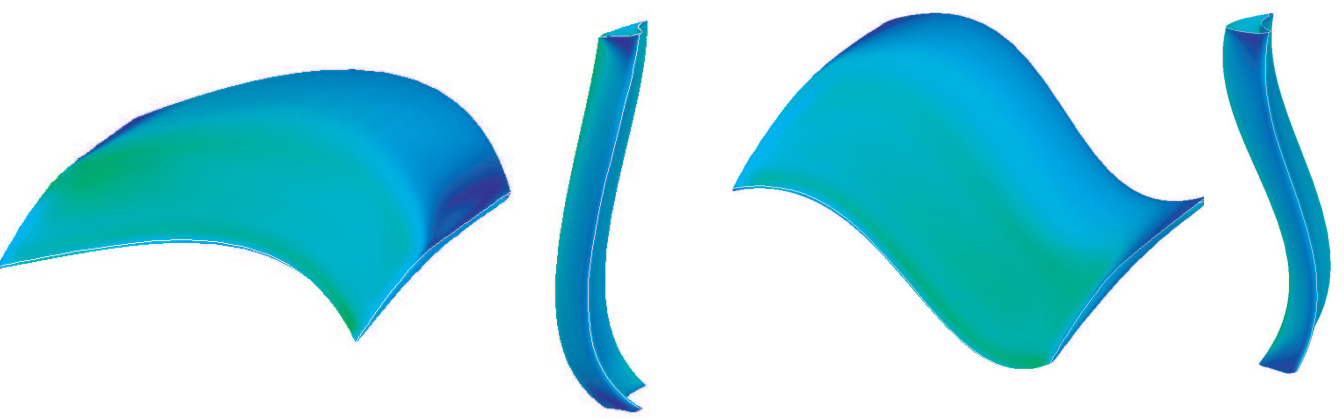

Three-objective compromise optimum design

Four-objective compromise optimum design

Figure 1. Optimum blade geometries found by different optimisation approaches
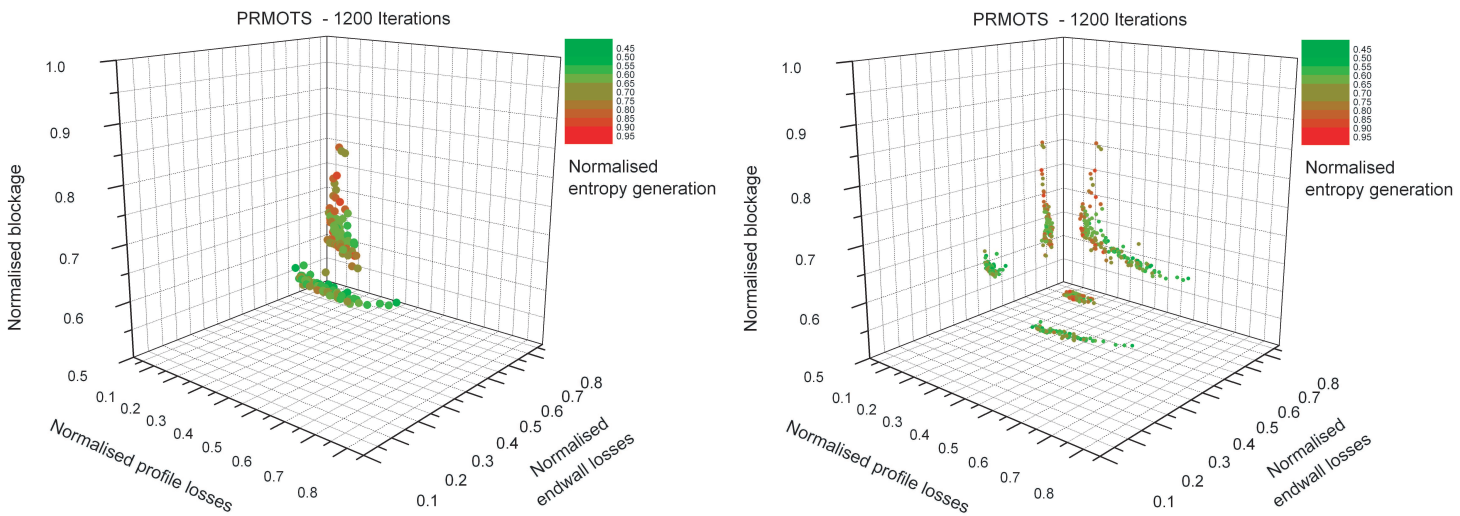

Figure 2. The 4D Pareto front found using PRMOTS

The behaviour of PCMOTS in our 3D aerodynamic design case was similar to the one already shown by Ghisu et al. ${ }^{6] \mid 9]}$ The use of the technique reduced the dimensionality of the design space significantly, improving the quality of the Pareto front. In particular, significantly reduced values of normalised endwall losses were found; the values of normalised blockage were reduced noticeably, while the region of minimum normalised profile losses was perhaps not similarly well explored. Figure 3 presents the Pareto front found with PCMOTS and the differences compared to the trade-off found with PRMOTS, Fig. 2, are clear, especially for the normalised endwall losses.

In an effort to facilitate a better exploration of the trade-off surface we modified the Intensification Memory (IM) management of the PCMOTS algorithm. We define an upper limit on the size of the IM 

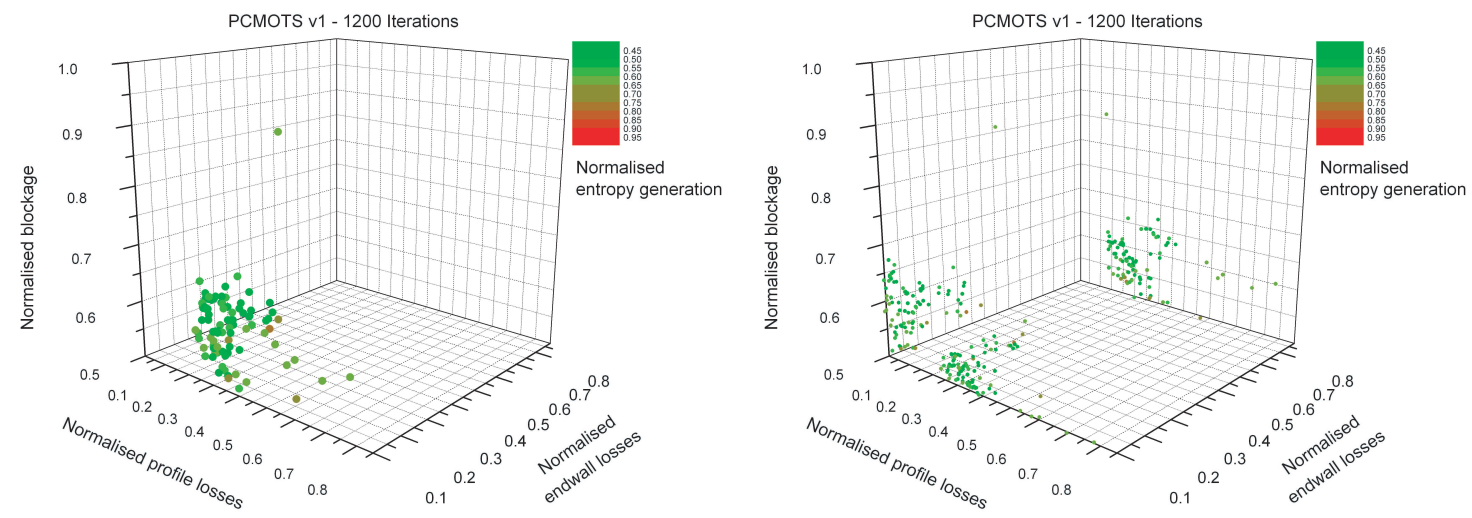

Figure 3. The 4D Pareto front found using PCMOTS
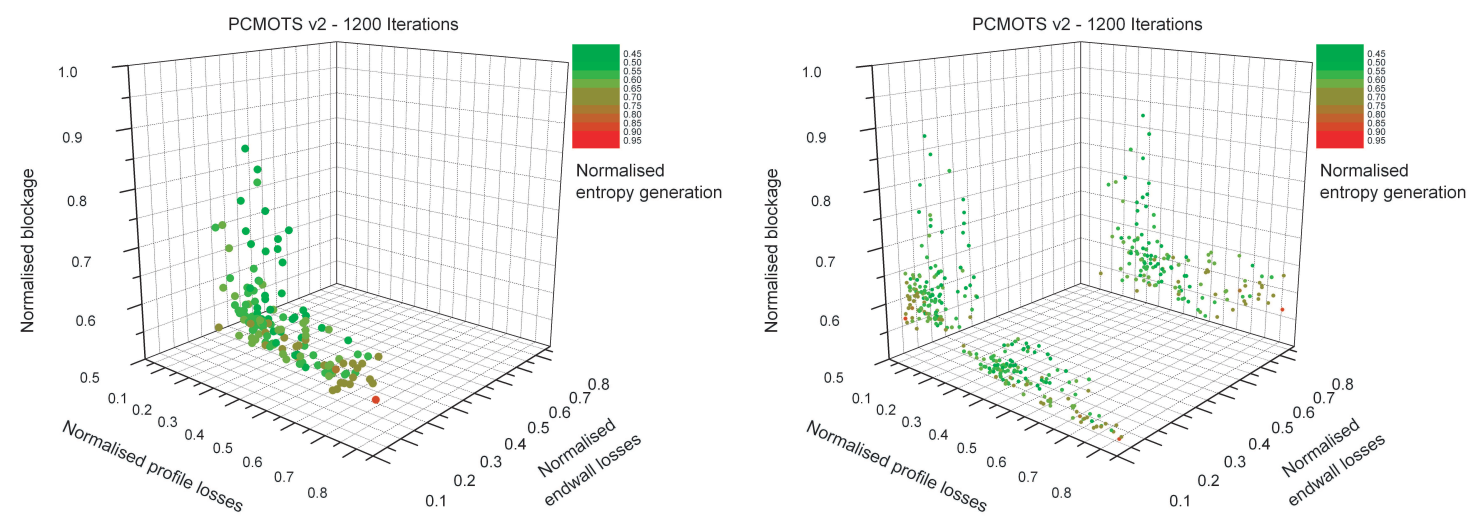

Figure 4. The 4D Pareto front found using enhanced PCMOTS

and we include the Pareto-optimal designs that maximise the coverage of the actual Pareto-front (this is achieved by selecting a random design from the Pareto front first, and then adding the point that maximises the mean distance from the already selected individuals, until the limit for the IM size is reached). We also include all the extremes of the current Pareto front (the solutions with the lowest value of each individual objective). When intensification occurs it is quite likely to relocate the search to an extreme solution and, in this way, we prioritise exploration of the ends of trade-off surface, as well as minimising the risk of a repeated exploration of an already well-explored region of the trade-off surface.

Figure 4 illustrates the trade-off found with this second variant of PCMOTS. It is evident that all of the extremes were explored thoroughly; at the same time, the number of Pareto-optimal solutions is larger, meaning that the diversification of the IM has worked satisfactorily.

It should be highlighted that, while all these runs were executed with the same number of TS iterations, in both cases PCMOTS required 25\% fewer objective function evaluations than PRMOTS, with an important advantage in terms of computational cost.

In order to assess the actual optimisation performance of each algorithm we need to assign a measure of quality to the revealed Pareto fronts. Two of the most common performance criteria for multi-objective optimisation are the unary epsilon indicator and the hypervolume indicator. The former criteria was proposed by Zitzler et al $\stackrel{22]}{[2}$ and the latter by Zitzler and Thiele, 21 and both are discussed in depth in. 22

Due to the metaheuristic nature of the TS algorithm, optimisation runs are not deterministic. Performance assessments of metaheuristic (or stochastic) optimisers require multiple runs of the same optimisation case, in order to minimise the dependence of the performance metric on the stochastic elements of the search. However, due to the computational cost for each optimisation run (about a week) we executed only two optimisations with each variant of the MOTS implementation. This operation produced 6 four-dimensional trade-off surfaces, and the combined Pareto Set among these was assigned as the reference optimal set. The 
mean and standard deviation values for the epsilon and hypervolume indicators are presented in Table2 for each algorithm.

Table 2. Means and Standard Deviations of the hypervolume and epsilon performance indicators

\begin{tabular}{lllllll}
\hline Indicator & PRMOTS & & PCMOTS v1 & \multicolumn{2}{c}{ PCMOTS v2 } \\
\hline & Mean & SD & Mean & SD & Mean & SD \\
\hline hyp & 0.5281 & 0.1166 & 0.1190 & 0.0786 & 0.1968 & 0.0211 \\
eps & 0.4484 & 0.1447 & 0.1270 & 0.0726 & 0.1422 & 0.0147 \\
\hline
\end{tabular}

When an individual Pareto front is assessed with respect to the reference trade-off surface, these indicators should take a minimum value, in order to express high optimum quality. According to this definition and observing the mean values as presented in Table 2, both variants of PCMOTS outperform PRMOTS. The stadard deviation is also reduced, and this is an indicator of a more consistent behaviour. The first PCMOTS variant produces slightly lower values for the indicators than the second. The number of Pareto optimal solutions found by the three variants is presented in Table 3. It is clear that the improved values found by the PCMOTS variants were obtained at the price of a reduced richness of the trade-off surface. The two runs for the second variant of PCMOTS were obtained with different limits on the IM size (5 and 10, respectively). It seems that a small limit on the IM size produces a better behaviour for the algorithm.

Table 3. The size of the Pareto Sets revealed from each variant of MOTS

\begin{tabular}{llll}
\hline Optimisation run & PRMOTS & PCMOTS v1 & PCMOTS v2 \\
\hline 1 & 164 & 91 & 140 \\
2 & 133 & 106 & 56 \\
\hline
\end{tabular}

Another interesting comparison comes from the percentage of the reference set (which is an overall Paretofront from the combination of all available runs) as generated by the different MOTS variants. These results are reported in Table 4. It is clear how PCMOTS is able to cover a significantly larger portion of the "true" Pareto-front (the one taken as reference for this analysis), while also reducing the computational cost.

Table 4. Percentage of the reference set produced by each variant of MOTS

\begin{tabular}{lll}
\hline PRMOTS & PCMOTS v1 & PCMOTS v2 \\
\hline $1.54 \%$ & $85.38 \%$ & $13.08 \%$ \\
\hline
\end{tabular}

\section{Analysis of the Design Space in Parallel Coordinates}

When performing real-world shape optimisation design studies it is really important to analyse the optimum design configurations, in order to relate geometrical characteristics with optimum behaviour of the objective function. Such analysis is possible to happen when we are able to visualise the whole design parameter space together with the objective function space. It is clear that the dimensionality of such datasets is quite high, as it is in the case we are presenting in this paper ( 26 design parameters +4 objective functions). However, we can easily represent this hyper-space in Parallel Coordinates ${ }^{12}$ and there is a methodology, as described by Kipouros et al.,17 that we can extract such qualitative information.

Figures 5 and 6 illustrate the Parallel Coordinates analysis for representative trade-off surfaces revealed with PRMOTS and PCMOTS respectively. Dimensions $x 1$ to $x 26$ express the design parameters and $c 1$ to $c 4$ the objective functions of the optimisation problem; profile losses, endwall losses, blockage, and entropy generation rate respectively. It is clear that the most influencial design parameters have been identified better with PCMOTS, and hence, the significant reduction to the dimensionality of the design problem. There are

$$
6 \text { of } 9
$$

American Institute of Aeronautics and Astronautics 

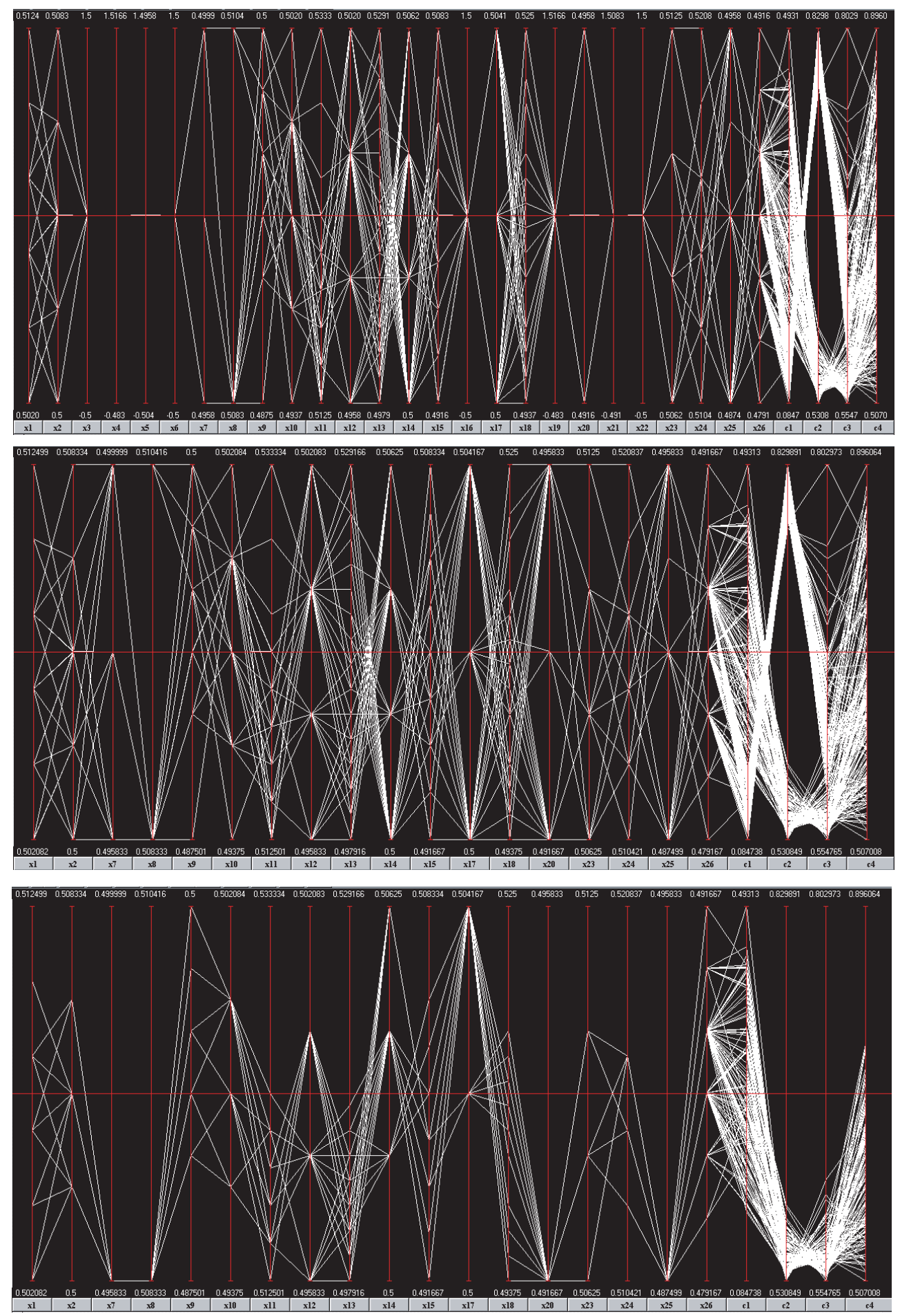

Figure 5. Analysis and visualisation in Parallel Coordinates of the Pareto front revealed with PRMOTS (Full dataset - Full dataset without the contant parameters - An identified pattern)

18 active design parameters within the PRMOTS Pareto Set, and only 13 for the PCMOTS trade-off surface.

However, for this case (Fig 6 ) there is not a clear pattern that relates design parameters with objective functions. In contrast though, in Fig 5 design parameters $x 20$ and $x 25$ express the whole optimum region for lowest $c 2$ and $c 3$ objective functions. This is a very important observation that assists towards the physical understanding of the optimality level of the operation of compressor blades. 

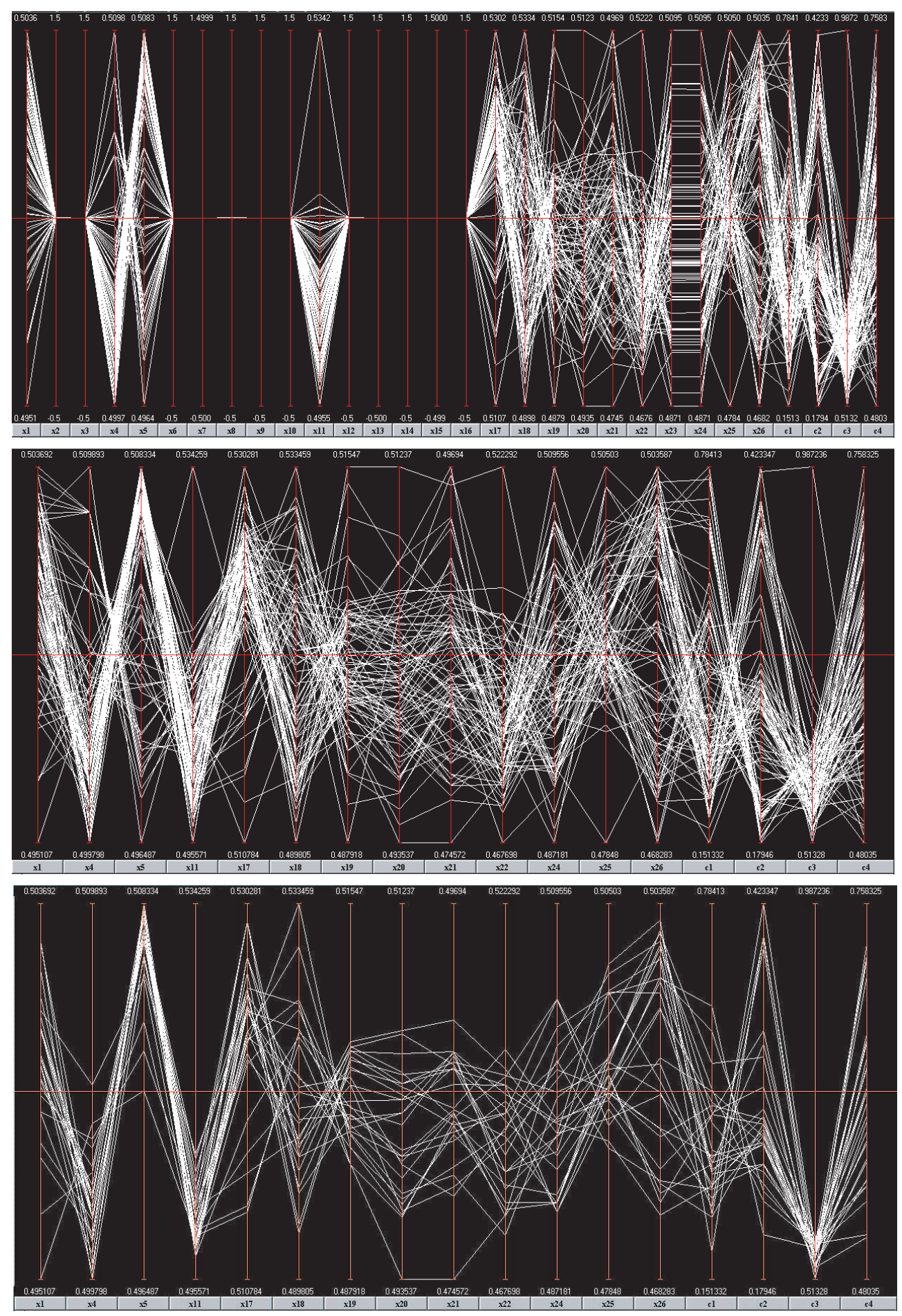

Figure 6. Analysis and visualisation in Parallel Coordinates of the Pareto front revealed with PCMOTS (Full dataset - Full dataset without the contant parameters - An identified pattern)

\section{Conclusions}

It has been shown that PCMOTS is more efficient for rapid design space exploration. Being able to identify the most energetic directions of the design space, the PCMOTS variant is able to explore the design space more quickly, producing better designs in a reduced time. Previously, these results had been demostrated both through mathematical test functions and in a real-world aerodynamic design problem (the 
preliminary design of a core compression system). Our test case represents a potentially more challenging problem, as detailed aerodynamic optimisation (using three-dimensional CFD) is likely to generate a more fragmented and discontinuous design landscape. Even in this case, PCMOTS outperformed the original PRMOTS implementation. The improved performance was demonstrated by the lower values of epsilon and hypervolume indicators, as well as the capability to cover a significantly larger portion of the reference Pareto front, while also reducing the computational cost (lower number of evaluations) by about $25 \%$.

With a modified implemetation of the Intensification Memory (IM) of the PCMOTS algorithm we can rapidly explore complicated design spaces and also identify the extreme optimum areas for each objective function. At the same time though the Pareto front is not very rich, representing incomplete exploration of the compromise optimum design area that penalises the quality of the Pareto Set. It is anticipated that with proper tuning of the IM size parameter PCMOTS will reveal the same quality trade-off surfaces as these revealed with PRMOTS, but at considerable reduced computational cost. This characteristic is extremely important for real-world computational engineering design problems, in particular when robustness criteria are considered during the design process.

Further investigations towards the balance of efficiency and quality of optimisation algorithms will be proved extremely beneficial in computational engineering design.

\section{References}

\footnotetext{
${ }^{1}$ Bloor, M. I. G., Wilson, M. J.: Efficient Parameterisation of Generic Aircraft Geometry. Journal of Aircraft. 32 (1995) $1269-1275$

${ }^{2}$ Deb, K., Thiele, L., Laumanns, M., and Zitzler, E., 2002. Scalable multi-objective optimization test problems. In: Congress on Evolutionary Computation (CEC2002), 825-830.

${ }^{3}$ Epstein, B., Peigin, S.: Constrained Aerodynamic Optimization of Three-Dimensional Wings Driven by Navier-Stokes Computations. AIAA Journal. 43 (2005) 1946-1957

${ }^{4}$ Gaiddon, A., Knight, D. D., Poloni, C.: Multicriteria Design Optimisation of a Supersonic Inlet Based upon Global Missile Performance. Journal of Propulsion and Power. 20 (2004) 542-558

${ }^{5}$ Ghisu, T., Parks, G. T., Jarrett, J. P., and Clarkson, P. J., Robust Design Optimization of Gas Turbine Compression Systems, Journal of Propulsion and Power, 27(2) (2011) 282-295

${ }^{6}$ Ghisu, T., Parks, G. T., Jaeggi, D. M., Jarrett, J. P., Clarkson, P. J.: The Benefits of Adaptive Parametrization in Multi-objective Tabu Search Optimization. Engineering Optimization. 42 (2010) 959-981

${ }^{7}$ Ghisu, T., Parks, G. T., Jarrett, J. P., Clarkson, P. J.: Accelerating Design Optimisation via Principal Components Analysis. AIAA Paper 2008-5855 (2008)

${ }^{8}$ Ghisu, T., Parks, G. T., Jarrett, J. P., and Clarkson, P. J., An Integrated System for the Aerodynamic Design of Compression Systems - Part I: Development ASME Journal of Turbomachinery, Vol. 133, No. 1, 2011, pp. 011011-1-01101110 .

${ }^{9}$ Ghisu, T., Parks, G. T., Jarrett, J. P., and Clarkson, P. J., An Integrated System for the Aerodynamic Design of Compression Systems - Part II: Application ASME Journal of Turbomachinery, Vol. 133, No. 1, 2011, pp. 011012-1-011012-8. ${ }^{10}$ Glover, F., Laguna, M.: Tabu Search. Kluwer Academic Publishers, Boston MA (1997)

${ }^{11}$ Harvey, S. A., Dawes, W. N., Gallimore, S. J.: An Automatic Design Optimisation System for Axial Compressors, Part I: Software Development. ASME GT2003-38115 (2003)

${ }^{12}$ Inselberg, A.: Parallel Coordinates: Visual Multidimensional Geometry and its Applications. Springer, New York (2009)

${ }^{13}$ Jaeggi, D. M., Parks, G. T., Kipouros, T., Clarkson, P. J.: The Development of a Multi-Objective Tabu Search Algorithm for Continuous Optimisation Problems. European Journal of Operational Research. 185 (2008) 1192-1212

${ }^{14}$ Kipouros, T., Jaeggi, D. M., Dawes, W. N., Parks, G. T., Savill, A. M. Multi-criteria Optimisation of Turbomachinery Blades: Investigating the Trade-off Surface. AIAA Paper 2005-4023 (2005)

${ }^{15}$ Kipouros, T., Jaeggi, D. M., Dawes, W. N., Parks, G. T., Savill, A. M., Clarkson, P. J. Biobjective Design Optimization for Axial Compressors Using Tabu Search. AIAA Journal. 46 (2008) 701-711

${ }^{16}$ Kipouros, T., Jaeggi, D. M., Dawes, W. N., Parks, G. T., Savill, A. M., Clarkson, P. J. Insight Into High-quality Aerodynamic Design Spaces Through Multi-Objective Optimization. CMES: Computer Modeling in Engineering \& Sciences. 37 (2008) 1-23

${ }^{17}$ Kipouros, T., Mleczko, M., Savill, A. M. Use of Parallel Coordinates for Post-Analyses of Multi-Objective Aerodynamic Design Optimisation in Turbomachinery. AIAA Paper 2008-2138 (2008)

${ }^{18}$ Sasaki, D., Obayashi, S., Nakahashi, K.: Navier-Stokes Optimization of Supersonic Wings with Four Objectives Using Evolutionary Algorithm. Journal of Aircraft. 39 (2002) 621-629

${ }^{19}$ Shahpar, S.: Automatic Aerodynamic Design Optimisation of Turbomachinery Components - An Industrial Perspective. VKI lecture series on Optimisation Methods \& Tools for Multicriteria/Multidisciplinary Design (2004) 1-40

${ }^{20}$ Zachariadis, Z. I.: High Resolution and High Order Methods for RANS Modelling and Aerodynamic Optimization. Ph.D. Dissertation. University of Cranfield (2008)

${ }^{21}$ Zitzler, E., Thiele, L.: Multiobjective Optimization using Evolutionary Algorithms - a Comparative Study. In Proceedings of Parallel Problem Solving from Nature - PPSN V. LNCS 2632 (1998)

${ }^{22}$ Zitzler, E., Thiele, L., Laumanns, M., Fonseca, C. M., Grunert da Fonseca, V.: Performance Assessment of Multiobjective Optimizers: An Analysis and Review. IEEE Transactions on Evolutionary Computation. 7 (1998) 117-132
} 